\section{Introducing Oncoscience:}

\section{from Oncotarget to the future}

In May 2010, Oncotarget was launched. Just in a couple of years, Oncotarget has received its 2012 impact factor of 6.67 , becoming one of the leading journals in oncology and cancer research. The number of submissions is increasing faster than exponentially. Now it is a bi-weekly journal, with dozens of excellent research papers per issue. If this trend continues, Oncotarget may have pretty good odds to attract nearly all submissions in the field of cancer. Rapid success and increasing popularity of Oncotarget prompts us to launch Oncoscience, a sister journal on a broader range of topics, with a larger Editorial Board, potentially numerous sections headed by associate editors, and... ambitious goals.

One may ask why do we start Oncoscience, instead of allowing Oncotarget to grow up?

Oncotarget was envisioned as a small journal that introduced a new concept of oncotarget, promoting a multidisciplinary approach with a unique focus. And we wish to keep it without changes. In a way, Oncotarget was an experiment. The journal became very successful and popular. The key is the passion, simplicity, an individual approach, avoidance of unqualified reviewers, evaluation of the submission in parallel rather than in sequence and a full use of all technical possibilities that are provided by modern technology. Despite unprecedented technical progress of science and technology, the routine of the most journals remains unchanged. A new paradigm of the scientific publication is desperately wanted by the scientific community. Scientists must have an alternative to the traditional publication process, which sometimes consists of a year-long Russian roulette.

How to reconcile a thorough peer-review with rapid publication? How to make some key decisions on the day of submission? How to publish the next day after acceptance? How to increase the visibility of the published papers? This Editorial is not a manual on the revolutionary scientific publishing. So, only two examples. When a paper is submitted, it should go immediately to actual Editors (not only to managing staff), to actual editors' e-mails and cell phones. Unlike managing staff, which works from 9 to 5 (and do not work on Christmas), actual editors are curious enough to read a new submission even on Valentine's day. Outstanding papers can be sent to 3-5 reviewers in one hour after submission. This saves 2-3 weeks to start with. And it is also important that most papers are rejected at the day of submission, thus saving the authors time and efforts. Collaboration between editors and reviewers is essential. It is important that Editors reviewed the papers themselves too, to judge rebuttals. Non-qualified judges hurt the journal.

Another example is an individual approach. Consider a paper rejected from a famous journal (with impact factor 30), after two rounds of peer-review by 3 reviewers, two rounds of rebuttals and year-long additional experiments. In evaluation of such a paper, previous rebuttals and reviews are very valuable. Internal peer-review may be a key in such cases, and the paper can be published in two-nights after submission. Editors and authors have a common goal, the best possible publication. So informal interaction is also important. How to obtain reviews fast? How to avoid "bad" reviewers? Well, we have the answers but this Editorial is not a manual.

Finally, it is very important to be sympathetic to the authors. If rejected, the paper should be rejected fast. If authors are asked for additional experiments, the editor should be sure that the paper is potentially acceptable. And it is important to avoid unnecessary experiments just because the reviewer is curious. The authors should continue their research and work on the next paper. After all, journals serve the scientific community and, if some of them do not, the scientists will publish elsewhere. This is why authors choose Oncotarget and prompt us to extend it by starting Oncoscience. Oncoscience has been launched under the supervision of the editorial board of Oncotarget, which includes Nobel and Lasker Prize winners, many members of National Academy of Sciences USA, and the most cited authors. The board will be extended and the sections will be created.

Oncoscience is not intended as a second-rate journal to publish papers rejected by Oncotarget. In contrast, it is built on the achievements of Oncotarget, whose experiment has proved successful. Now we can be confident to launch a journal that can potentially become the main transmitter of scientific information in the field. 